\title{
Elderly man with confusion: the unexpected
}

\author{
Anver Sadath ${ }^{1}, J D V C_{\text {Lekamwasam }}{ }^{2}$, ES de Silva $^{3}$, SP Dissanayaka ${ }^{1}$ \\ ${ }^{I}$ Registrar in Medicine, ${ }^{2}$ Consultant Physician, ${ }^{3}$ Senior Registrar in Medicine, Teaching Hospital, \\ Karapitiya, Galle.
}

A 69 year-old diagnosed patient with hypertension and ischaemic heart disease was admitted to hospital with a four day history of dysurea, vomiting, reduced urine output and altered behaviour for one day.

He was well until one month prior to the hospital admission when he developed an upper respiratory tract infection which lasted for three days and on the fourth day of the illness he became confused together with an unsteady gait. He was admitted to a district general hospital where he was infused with intravenous fluids to which there was a dramatic response. He was also found to be anaemic and the blood picture was normochromic, normocytic but a cause for the anaemia was not established. He was transfused with a pint of blood and discharged.

He was well for three weeks until he was admitted to the Teaching Hospital, Karapitiya with symptoms suggestive of a urinary tract infection. On examination he was afebrile, confused, pale and hypotensive. Reduced body hair was also noted. There were no focal neurological signs.

On admission the following differential diagnoses were considered; urinary tract infection, electrolyte imbalance secondary to vomiting and chronic subdural haemorrhage.

Treatment was commenced empirically with intravenous Co-amoxyclav. Investigations revealed the following: UFR - normal, Urine culture - sterile, $\mathrm{Na}^{+}-125 \mathrm{mmol} / \mathrm{L}, \mathrm{K}^{+}-6$ $\mathrm{mmol} / \mathrm{L}, \mathrm{RBS}-3.3 \mathrm{mmol} / \mathrm{L}, \mathrm{Hb}-11 \mathrm{~g} / \mathrm{dL}$ and
Chest X-Ray - normal. Patient remained confused despite treatment. There was persistent hyperkalaemia with hyponatraemia and the blood pressure remained low which prompted us to investigate for a possible hypoadrenalism. He was started on intravenous dexamethasone and normal saline for which he showed a marked improvement. Subsequent investigations revealed a low ACTH concentration $(<10 \mathrm{pg} / \mathrm{mL}$ - mean $24 \mathrm{pg} / \mathrm{mL}$ ) with a low 6.00 a.m. cortisol concentration $(35.8 \mathrm{nmol} / \mathrm{L}$ - Normal range - 100 $-600 \mathrm{nmol} / \mathrm{L}$ ) confirming our tentative diagnosis of secondary adrenal insufficiency. CT scan of the brain revealed an intrasellar pituitary tumour. On further evaluation of the pituitary hormone profile a low concentration of LH $(0.79 \mathrm{u} / \mathrm{L}$ Normal range1-10 u/L) and a low normal concentration of GH ( $4.35 \mathrm{u} / \mathrm{L}$ - Normal range > $4 \mathrm{u} / \mathrm{L})$ was found. Rest of the anterior pituitary hormone concentrations remained within normal range. Since the tumour showed mass effect he was referred for neurosurgical management.

\section{Discussion}

Pituitary tumours are uncommon (1-2/100,000 patients per year) [1]. They account for $10 \%-$ $15 \%$ of intracranial tumours and $75 \%$ of them secrete inappropriate levels of pituitary hormones [2]. Almost all the pituitary tumours are benign [1]. Typical presenting features include hormonal hypersecretion, visual field defects, headache, hypopituitarism, pituitary apoplexy, hydrochephalus, cranial nerve palsies and temporal lobe epilepsy. 


\section{Case Reports}

This patient presented with anaemia and recurrent episodes of confusion which could have occurred due to adrenal insufficiency precipitated by trivial infection possibly a respiratory tract infection or urinary tract infection.

This case demonstrates the need to be vigilant about the possibility of rare conditions presenting as common clinical presentations like anaemia and confusion in the elderly.

\section{References}

1. David A warrell, Timothy M Cox, In: Oxford Text Book of Medicine, Oxford University press, $4^{\text {th }}$ edition, 2003; 2: 192-5.

2. A Levy, S. L. Lightman. Diagnosis and management of pituitary tumours, British Medical Journal, 1994 308:1087-91. 